\title{
Compound-Fault Diagnosis of rolling bearing based on Order Wavelet Packet and Rough Sets Theory
}

\author{
KANG Hai-ying $^{1, a}$, QI Yan-jie ${ }^{2, a}$, LIU Guang-sheng ${ }^{1, a}$, SHEN Ren-fa ${ }^{1, a}$ \\ ${ }^{1}$ Department of Guns Engineering, Ordnance Engineering College, Shijiazhuang, 050003; \\ 2 Dept. of Basic Courses, Xuzhou Air Force College, Xuzhou, 221000, China \\ a E-mail: hykangqi@163.com
}

\begin{abstract}
Keyword: order wavelet packet, rough sets theory, bearing, decision rule, compound-fault diagnosis
\end{abstract}

\begin{abstract}
The diagnosis of compound-fault is always a difficult point, and there is not an effective method in equipment diagnosis field, and then a new method of compound-fault diagnosis was presented in the paper. The vibration signals at start-up in the gearbox are non-stationary signals, in order to solve the compound-fault diagnosis of rolling bearing, the order tracking wavelet packet and rough sets theory are combined and introduced into the fault diagnosis field. Firstly, the non-stationary vibration signals at start-up were resampled using computer order tracking arithmetic and equal angle distributed vibration signals were obtained, and then the signals were decomposed and recomposed with the wavelet packet. The energy distribution of every frequency band was calculated according to normalization process. A new feature vector can be obtained, then clear and concise decision rules can be obtained by rough sets theory. Finally, the result of compound-fault example proves that the proposed method has high validity and more amplitude appliance foreground.
\end{abstract}

\section{Introduction}

The rolling bearing is one kind of equipment, which is extensive in existence range, most amount and applied in most universal in many machine. When there are fault in rolling bearing, the natural frequency of bearing vibration system will be activated by high-frequency vibration, which is generated by local scathing component of rolling bearing at work [1]. It is very useful and necessary for researching the compound-fault of rolling bearing. The vibration signals of rolling bearing are non-stationary signals and it is consisted of cluster harmonic components of fault character frequency. There are quadratic phase angle couple in local dandify vibration signals of rolling bearing. The fault vibration signals of rolling bearing is non-stationary and non-gauss distribution signals [2]. Under this condition, the request of stationary about signals can't be suited to FFT. In order to solve the problem, the theory of angle sample and theory of order tracking analysis, which based on the theory of angle sample, are generated.

Compared with the normal signals, the energy of some frequency band lies more difference, it is may monish or accretion. So, the energy of the frequency band signals is included abundant fault information [4]. The wavelet analysis is one advanced measure of time-frequency analysis. The fault character of impulsion signals in vibration signals can be effectual distilled. It is one availability method for rotate machine fault character distilling. The rough set theory is one new mathematics tool which is disposed with the fussing mathematics and indetermination problem. When the information is disposed, the data is reduced and the least expression of intellective is obtained on the precondition of the key information is no dignify. The minimal rule is obtained. The aptitude fault diagnosis method based on rough set theory is the method based on information. This is the most excellence which is compare with the fuzzing theory and evidence theory [3]. So, it is very important that the rough set method of equipment fault diagnosis is developed.

In the field of equipment fault diagnosis, the diagnosis of compound-fault is always the difficulty [4]. The order wavelet change is adopted as the disposal tool of signals in the paper. The vibration signals during the course of start-up in gearbox is analyzed with order tracking, the signals after 
resampling in angle-domain are decomposed with wavelet, and then the character parameter is distilled. The fault diagnosis decision-making rule of bearing is obtained with rough set. At last, the validity of the decision-making rule is validated through complex fault example.

\section{Order Tracking Analysis}

The order tracking technique is one kind of vibration measure technique for rotate frequency instability machine. The vibration signals, which are conceded with the rotate speed, can be separated availably during the course of machine shift speed. At the same time, the signals, which are irrespective with the rotate speed, are controlled. The excellence of the method is obvious for the shift rotate speed of machine. The sampling distant is distributed with turn angle station, and then the infection of frequency spectrum chart is eliminated for the rotate shift. The accuracy of vibration measure is pledged by the method, which the sampling frequency is advanced following the rotate speed hoist. When the rotate speed is high, the change of vibration wave is more acuity. The sampling point is encrypting folling enhancing the sampling frequency. The character points in vibration signals can be avoid lose.

The key of order tracking is how to realize the constant angle increment ( $\Delta \theta)$ sampling relative to consult shaft axle. The vibration signals are requested with synchronization sampling in accretes order tracking. The definition and dependability of monitoring system is laid on the quality of synchronization sampling. Computed Order Tracking (COT) is more agility compare with the traditional method, and the precision is the same as or more better the traditional ones. The most excellence lies that there is no need the specifically hardware. It is very important for many application of estate measure. Compare with the traditional method, the cost and employ are predigested, and it is successful used in engineering practicality [3-5].

\section{The Theory of Wavelet Package Energy Spectrum analysis}

The fault signals of collection are digital signals. In order to realize wavelet decomposition for the digital signals, it can be supposed that $\left\{S_{k}, k \in N\right\}$ is disperse sequence, and the operator is defined,

$$
\begin{gathered}
F_{0}\left\{S_{k}\right\}_{(j)}=\sum_{k \in N} h_{k-2 j} S_{k} \\
F_{1}\left\{S_{k}\right\}_{(j)}=\sum_{k \in N} g_{k-2 j} S_{k}
\end{gathered}
$$

The $\left\{f_{p}(00), p \in N\right\}$ is supposed as disperse sequence of original fault signals, and then the arithmetic of wavelet decomposition is [1],

$$
\begin{array}{r}
f_{p}(2 n, j+1)=F_{0}\{f(n, j)\}_{(p)} \\
f_{p}(2 n+1, j+1)=F_{1}\{f(n, j)\}_{(p)}
\end{array}
$$

Where, $p$ is the each sequence point of wavelet decomposition. $j$ is the wavelet decomposition layer. After decomposition, the energy character value of each frequency channel signals is colligating all the fault information in time-domain and angle-domain. The signals energy of each frequency channel is analyzed with statistic analysis. The character vector is formed, which is reflecting the fault signals. The energy of each frequency channel is express as,

$$
E=\sum_{k=1}^{N}\left|S_{j k}\right|^{2}
$$

Where, $j$ is frequency multiplication wavelet decomposition layer, $N$ is the number of sampling signals.

If the signals are decomposed with wavelet, one character vector can be constructed with energy element. The construction of character vector $T$ is,

$$
T=\left[E_{j 0}, E_{j 1}, \cdots, E_{j m}\right]
$$


Where, $m=2^{j}-1$.

In order to compare the size of each frequency band energy, the energy vector is disposed with normalization, and then the energy character vector of fault signals are obtained. At last, the character vector of bearing fault is obtained.

\section{The Elementary Knowledge of Rough Set Theory}

At present, the application of fault diagnosis with the rough set is mostly as [6-7],

(A)The fault diagnosis character is distilled with the rough set theory and the each generalization model.

(B)The dimension of fault character input quantity is minished and the fault diagnosis character is simplified with the rough set. The dimensions and complexity of fault diagnosis system are reduced, and then the fault diagnosis mode of contracted is obtained.

(C)It can be application into the diagnosis system, which is combined with the other theory just as fuzzy logic, nerve network etc.

\section{A. Decision-making table and discrimination}

The knowledge system can be expediently expressed by using table of the database, and the attribute is denoted by arrange, and the record is denoted by row. The recording information is denoted by each row, each value in the table is the attribute value, and all of them are correspond the row and arrange. The decision-making table is one kind of special and important information expression system, most of the decision-making problem can be expressed by the decision-making table, it is important in the field of application decision-making. The knowledge is extracted from the decision table composed of consecutive value; firstly, the value area of condition attribute is needed to be discredited.

\section{B. Reduction of the decision-making table}

In the decision-making system, the attribute include condition attribute and decision attribute, and each condition attribute is not equal importance to the last decision, even some are redundancy. Especially the redundancy is more widespread when there is no explicit purpose. The existence of the redundancy attribute, on the one hand the saving space is enlarged, the difficulty of knowledge detection is increased. On the other hand, it is disadvantageous to make an accurate decision for people. The attribute reduction of the rough sets theory means to delete the attribute of the redundancy, which under the situation that keep a classification ability of make policy the system constant. The redundancy and useless component in database can be wiped off through attribute reduction, and the cryptic rule in data is open out. The nucleus is couldn't eliminated, which is the set of information character in the information reduction. For the reduction of nucleus attribute, it can be realized through solving the discernibility matrix.

\section{Fault Diagnosis Example of Bearing}

The examination is certificated in one kinds of single-stage gearbox. In the system, the input axis is driven by a speed-regulating electric motor; and the magnetic powder load is driven by the output axis. The number of teeth in drive gear is $Z 1=30$, the number of teeth in driven gear is $Z 2=50$. The model of rolling bearing in input is 206 . One small groove, which the width is $0.5 \mathrm{~mm}$ and deep is $1.5 \mathrm{~mm}$, is machining in the inner and outer of bearing to simulate the singleness fault of bearing inner crack and bearing outer crack. One small bit of a ball bearing is detruncated with linear cutting method to simulate the singleness fault of ball bearing. Three kinds of compound-fault are obtained through assemblage the three singleness fault. It is the bearing outer and ball bearing fault, the bearing inner and outer fault, the bearing inner and ball bearing fault.

The raising speed course of gearbox is analyzed; the rotate speed of input-axis is accelerated from 0 to 1200rpm. The vibration signals with B\&K4508 acceleration transducer and speed signals with JN338A rotary rotational speed and torque-admeasuring apparatus are delivered to B\&K4508 multi-analyzer for data processing. The vibration signals and rotate speed signals are sampled at constant time increments, the frequency of sampling is $16384 \mathrm{~Hz}$; the sampling time is $2 \mathrm{~s}$. The 
vibration signals in various work condition are extracted 30 group data, the 20 group data are used to train data, the other 10 group data are used to test data.

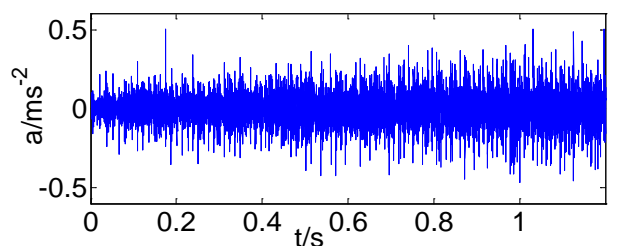

Fig. 1 the original vibration signsls in time-domain

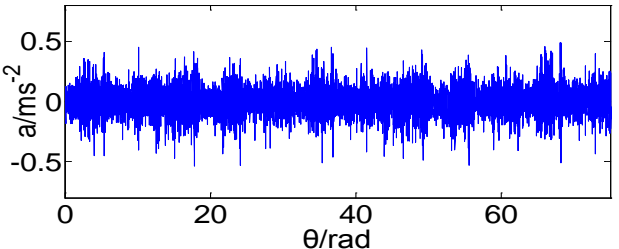

Fig.2 Vibration signals in angle-domain

Fig. 1 is original vibration signals in time-domain during the bearing inner and ball bearing compound-fault. It can be seen form the Fig. 1 that the amplitude of vibration signals is changed following the fluctuate of rotate speed. There are direct connection between the vibration signals in gearbox and the rotate speed of input axis. It is one obvious nonstationary course, and it couldn't satisfy with the placidity request of FFT. The signals in time-domain must be resampled in angle-domain. Fig.2 is the vibration signals after resampled. Compared with the original signals, the stationary of the signals in angle-domain is more improvement, and it can fit the request of the FFT. It can be drawn through the calculation.

The operating mode of compound-fault in rolling bearing is just as the research object, the three kinds of compound-fault state of bearing are analyzed. Three groups of training stylebook are distilled from 20 groups of the waiting training stylebook. The domain $U=\{1,2,3,4,5,6,7,8,9\}$ is obtained. The the compound-fault signals in angle-domain are decomposed in two floor wavelet with $\mathrm{db} 1$ wave. The 4 normalization eigenvectors are obtained.

Table.1 Training stylebook data

\begin{tabular}{cccccc}
\hline $\mathrm{U}$ & $\mathrm{a}$ & $\mathrm{b}$ & $\mathrm{c}$ & $\mathrm{d}$ & $\mathrm{D}$ \\
\hline 1 & 0.1357 & 0.1877 & 0.9320 & 0.2787 & 1 \\
2 & 0.1306 & 0.1870 & 0.9345 & 0.2733 & 1 \\
3 & 0.1995 & 0.2873 & 0.7151 & 0.6052 & 2 \\
4 & 0.2081 & 0.2921 & 0.7251 & 0.5878 & 2 \\
5 & 0.5638 & 0.6299 & 0.4402 & 0.2937 & 3 \\
6 & 0.6382 & 0.5925 & 0.3863 & 0.3041 & 3 \\
7 & 0.1423 & 0.1920 & 0.9286 & 0.2837 & 1 \\
8 & 0.2115 & 0.2963 & 0.6893 & 0.6264 & 2 \\
9 & 0.6052 & 0.6309 & 0.3957 & 0.2813 & 3
\end{tabular}

$T^{\prime}=\left[E_{20} / E, E_{21} / E, \cdots, \overline{\left.E_{23} / E\right]}\right.$, the 4 eigenvectors is just as condition attribute, and expressed with a,b,c,d. The operating mode of rolling bearing is just as decision-making attribute, and expressed with D. In the data, 1 is expressed the compound-fault state of bearing outer and ball bearing, 2 is expressed the compound-fault state of bearing inner and ball bearing, 3 is expressed the compound-fault state of bearing inner and outer. The training stylebook data of rolling bearing, just as table.1, is established with the characteristic parameter of distilling.

The rough set can only disposed the dates of disperse attribute. The data of original compound-fault attribute is continuous, so the data must be disposed into discrimination. The discrimination method of equidistant is adopted in the paper, and the data discrimination outcome, just as table.2, is obtained.

Table.2 Data discrimination outcome

\begin{tabular}{cccccc}
\hline $\mathrm{U}$ & $\mathrm{a}$ & $\mathrm{b}$ & $\mathrm{c}$ & $\mathrm{d}$ & $\mathrm{D}$ \\
\hline 1 & 1 & 1 & 3 & 1 & 1 \\
2 & 1 & 1 & 3 & 1 & 1 \\
3 & 1 & 1 & 2 & 3 & 2 \\
4 & 1 & 1 & 2 & 3 & 2 \\
5 & 3 & 3 & 1 & 1 & 3 \\
6 & 3 & 3 & 1 & 1 & 3 \\
7 & 1 & 1 & 3 & 1 & 1 \\
8 & 1 & 1 & 2 & 3 & 2 \\
\hline
\end{tabular}


The table. 2 is processed attribute reduction with discrimination matrix arithmetic, and the discrimination matrix is obtained, just as the table.3 shows.

Table.3 Discrimination matrix C9x9

\begin{tabular}{|l|l|l|l|l|l|l|l|l|}
\hline$\varnothing$ & $\varnothing$ & cd & cd & abc & abc & $\varnothing$ & cd & abc \\
\hline$\varnothing$ & $\varnothing$ & cd & cd & abc & abc & $\varnothing$ & cd & abc \\
\hline$\varnothing$ & $\varnothing$ & $\varnothing$ & $\varnothing$ & abcd & abcd & cd & $\varnothing$ & abcd \\
\hline$\varnothing$ & $\varnothing$ & $\varnothing$ & $\varnothing$ & abcd & abcd & cd & $\varnothing$ & abcd \\
\hline$\varnothing$ & $\varnothing$ & $\varnothing$ & $\varnothing$ & $\varnothing$ & $\varnothing$ & abc & abcd & $\varnothing$ \\
\hline$\varnothing$ & $\varnothing$ & $\varnothing$ & $\varnothing$ & $\varnothing$ & $\varnothing$ & abc & abcd & $\varnothing$ \\
\hline$\varnothing$ & $\varnothing$ & $\varnothing$ & $\varnothing$ & $\varnothing$ & $\varnothing$ & $\varnothing$ & $\varnothing$ & abc \\
\hline$\varnothing$ & $\varnothing$ & $\varnothing$ & $\varnothing$ & $\varnothing$ & $\varnothing$ & $\varnothing$ & $\varnothing$ & abcd \\
\hline$\varnothing$ & $\varnothing$ & $\varnothing$ & $\varnothing$ & $\varnothing$ & $\varnothing$ & $\varnothing$ & $\varnothing$ & $\varnothing$ \\
\hline
\end{tabular}

It can be seen from the table.3, the nucleus attribute is c and d, the least reduction subset is (a,c,d) and (b,c,d), these data is corresponding the 2 kinds of finally decision-making table. In the paper, the reduction (a,c,d) is choose as decision-making table. The row of repeated are eliminated, just as the table. 4 shows.

Table.4 Reduction (a,c,d) corresponding the decision-making table

\begin{tabular}{ccccc}
\hline $\mathrm{U}$ & $\mathrm{a}$ & $\mathrm{c}$ & $\mathrm{d}$ & $\mathrm{D}$ \\
\hline 1 & 1 & 3 & 1 & 1 \\
3 & 1 & 2 & 3 & 2 \\
5 & 3 & 1 & 1 & 3
\end{tabular}

In order to validate the affectivity of the algorithmic, the four stylebook data of rolling bearing compound-fault are extracted, just as table 5.

Table.5 Diagnosis syletbook data

\begin{tabular}{rrrrrr}
\hline $\mathrm{U}$ & $\mathrm{a}$ & $\mathrm{b}$ & $\mathrm{c}$ & $\mathrm{d}$ & $\mathrm{D}$ \\
\hline 1 & 0.2096 & 0.2949 & 0.7304 & 0.5793 & 2 \\
2 & 0.1308 & 0.1827 & 0.9379 & 0.2642 & 1 \\
3 & 0.6131 & 0.6194 & 0.3991 & 0.2850 & 3 \\
4 & 0.2081 & 0.2937 & 0.7100 & 0.6053 & 2 \\
\hline
\end{tabular}

The record one in table 5 is diagnosed, the new training stylebook data is formed through collecting the first line data with the table 1 . The new data are dispersed, and then the new disperse outcome table is observed. It can be seen that the discrimination value of condition attribute a,b,c is $1,2,3$, which is corresponding to coding 10 . It can be draw the conclusion that the register one is corresponding to the estate bearing inner and ball bearing compound-fault from the second diagnosis rule in table 4 , and the estimation outcome is right. In the same argument, it can be estimated that the register two and three and four are difference corresponding to the circulation estate bearing outer and ball bearing compound-fault, bearing inner and outer compound-fault, bearing inner and ball bearing compound-fault, and the estimation outcomes are all right.

\section{Conclusion}

(A) During the shift gears drive in gearbox is analyzed, the phenomenon 'frequency smear', which is couldn't overcame with the traditional spectrum analysis, can be solved with order tracking analysis, and the nonstationary signals in time-domain are transformed into the stationary ones in angle-domain.

(B) The wavelet is the useful tools, which is analyzed the non-gauss and non-linearity signals, and the fault information is distilled effectively with the order wavelet analysis.

(C) The information reduction can be processed with the rough set theory; the redundancy attribute and the property value during the course of fault diagnosis are eliminated. The distinct and concise diagnosis decision-making rule is obtained. 
(D) The new method in the paper can be used in the field compound-fault about rotate machine, the localization, which is couldn't solved with the traditional diagnosis method, is solved with the new method. It has very extensive application value in practicality.

\section{ACKNOWLEDGMENT}

The work is supported by national natural science foundation of china (51175508 and 50775219) and national natural science foundation of hebei province (E2012506009) and college foundation of Ordnance Engineering College (YJJXM13009)

\section{REFERENCES}

[1] ZHANG Han-lei, ZHOU Jie-min, LI Gang. Mixed Fault Diagnosis Based on Wavelet Analysis in Induction Motors [J].Proceedings of the CSEE, 2006, 26 (8):159-162.

[2] Feng Zhipeng, Song Xigeng, Xue Dongxin. Survey of Vibration Fault Diagnosis of Rotational Machinery [J].Journal of Vibration and Shock, 2001, 20 (4):36-39.

[3] ZHU Jumei. The analysis of non-stationary vibration signals (serialize). JOURNAL OF VIBRATION AND SHOCK, Vol19 No. 2 2000, P87-90.

[4] FYFE K R,MUNCKEDS.Analysis of computed order tracking[J].Mechanical Systems and Signal Processing,1997,11(2):187-205.

[5] GUO Yu, Qin Shuren. Pseudo-speed tracking order analysis for non-stationary vibration signals of rotating machinery. JOURNAL OF VIBRATION AND SHOCK, Vol23 No. 1 2004, P61-66.

[6] Nowicki R,Slowinski R,Stefanowski J.Evaluation of vibroacoustic diagnostic symptoms by means of the rough sets theory. Computers in industry [J], 1992, 20(2):142-152.

[7] Lixiang Shen, Francis EH Tay, Liangsheng Qu, Yudi Shen. Fault diagnosis using rough sets theory.Computers in Industry [J].2000, Vo1. (43):61-72. 